\title{
Constant cycle and co-isotropic subvarieties in a Mukai system
}

\author{
Isabell Hellmann ${ }^{1}$
}

Received: 14 October 2020 / Accepted: 21 September 2021 / Published online: 10 November 2021

(c) The Author(s) 2021

\begin{abstract}
Combining theorems of Voisin and Marian, Shen, Yin and Zhao, we compute the dimensions of the orbits under rational equivalence in the Mukai system of rank two and genus two. We produce several examples of algebraically coisotropic and constant cycle subvarieties.
\end{abstract}

Keywords Birational geometry $\cdot$ Moduli spaces $\cdot$ Irreducilbe symplectic varieties

Mathematics Subject Classification $14 \mathrm{~J} 42 \cdot 14 \mathrm{C} 05 \cdot 14 \mathrm{M} 22 \cdot 14 \mathrm{C} 99$

\section{Introduction}

By a theorem of Beauville and Voisin [4], any point lying on a rational curve in a $\mathrm{K} 3$ surface $S$, determines the same zero cycle of degree one

$$
c_{S} \in \mathrm{CH}_{0}(S)
$$

called the Beauville-Voisin class. This class has the striking property that the image of the intersection product

$$
\operatorname{Pic}(S) \otimes \operatorname{Pic}(S) \rightarrow \mathrm{CH}_{0}(S)
$$

and $c_{2}(S)$ are contained in $\mathbb{Z} \cdot c_{S}$. Irreducible holomorphic symplectic manifolds are (one of the two classes of) higher dimensional analogs of K3 surfaces. It is expected that the Chow ring of an irreducible holomorphic symplectic manifold has a similar and particularly rich structure provided by the conjectural Bloch-Beilinson filtration and its conjectural splitting

Communicated by Herr Greb.

The author is supported by the SFB/TR 45 'Periods, Moduli Spaces and Arithmetic of Algebraic varieties' of the DFG (German Research Foundation) and the Bonn International Graduate School.

Isabell Hellmann

igb@math.uni-bonn.de

1 Mathematisches Institut, Universität Bonn, Endenicher Allee 60, 53115 Bonn, Germany 
[5]. In this context, Voisin introduced in [32] the notion of an algebraically coisotropic subvariety, which is an generalization of Lagrangian subvariety.

The goal of this note is to investigate the Chow group of zero cycles for the Mukai system of rank two and genus two. Specifically, we produce several examples of algebraically coisotropic subvarieties fibered into isotropic constant cycle subvarieties.

Let $(S, H)$ be a polarized K3 surface of genus 2, that is a double covering $\pi: S \rightarrow \mathbb{P}^{2}$ ramified over a sextic curve and $H=\pi^{*} \mathcal{O}(1)$ is primitive. We consider the moduli space $M=M_{H}(0,2 H, s)$ of $H$-Gieseker stable coherent sheaves on $S$ with Mukai vector $v=(0,2 H, s)$ where $s \equiv 1 \bmod 2$. This is an irreducible holomorphic symplectic variety, which is birational to the Hilbert scheme $S^{[5]}$ of five points on $S$. A point in $M_{H}(0,2 H, s)$ corresponds to a stable sheaf $\mathcal{E}$ on $S$ such that $\mathcal{E}$ is pure of dimension one with support in the linear system $|2 H|$ and $\chi(\mathcal{E})=s$. Taking the (Fitting) support defines a Lagrangian fibration

$$
f: M_{H}(0,2 H, s) \longrightarrow B:=|2 H| \cong \mathbb{P}^{5}
$$

known as the Mukai system of rank two and genus two [3, 23]. It enjoys many beautiful features and is studied from various perspectives. For example, one can view it as a compactified relative Jacobian, as a generalisation of the Hitchin system [11] or as the birational model of $S^{[5]}$ admitting a Lagrangian fibration.

For any irreducible, holomorphic symplectic manifold $X$ of dimension $2 n$, a brute force approach to finding constant cycle subvarieties (see Sect. 3.1 for the definition) is to consider the orbit under rational equivalence of a point $x \in X$. This is the countable union of algebraic subvarieties defined by

$$
O_{x}:=\left\{x^{\prime} \in X \mid[x]=\left[x^{\prime}\right] \in \mathrm{CH}_{0}(X)\right\} \subset X .
$$

Then $\operatorname{dim} O_{x}$ is defined to be the supremum over the dimensions of the components of $O_{x}$. In [32], Voisin defines an increasing filtration $F_{0} X \subset F_{1} X \subset \ldots \subset F_{n} X=X$ on the points of $X$, where

$$
F_{i} X:=\left\{x \in X \mid \operatorname{dim} O_{x} \geq n-i\right\}
$$

is again a countable union of algebraic subvarieties. Our examples are based on the combination of two theorems. The first one is due to Voisin.

Theorem 1.1 [32, Theorem 1.3] We have $\operatorname{dim} F_{i} X \leq n+i$ and if $Z \subset F_{i} X$ is an irreducible component of dimension $n+i$. Then $Z$ is algebraically coisotropic and the fibers of the isotropic fibration are constant cycle subvarieties of dimension $n-i$.

The second theorem applies in the case that $X=M_{\sigma}(v)$ is a smooth projective moduli of Bridgeland stable objects in $D^{b}(S)$ and is due to Marian, Shen, Yin and Zhao. It establishes a link between rational equivalence in $X$ and in $S$, which in particular results in a connection between Voisin's filtration $F . X$ and O'Grady's filtration $S . \mathrm{CH}_{0}(S)$ (see Sect. 3.1 for the definition). 
Theorem 1.2 ([29, Theorem 0.5], [22, Theorem], Theorems 3.2, 3.5)

(i) Any two points $\mathcal{E}, \mathcal{E}^{\prime} \in M_{\sigma}(v)$ are rational equivalent in $M_{\sigma}(v)$ if and only if $\operatorname{ch}_{2}\left(\mathcal{E}^{\prime}\right)=\operatorname{ch}_{2}(\mathcal{E}) \in \mathrm{CH}_{0}(S)$.

(ii) Let $\mathcal{E} \in M_{\sigma}(v)$ such that $\mathrm{ch}_{2}(\mathcal{E}) \in S_{i} \mathrm{CH}_{0}(S)$. Then $\mathcal{E} \in F_{i} M_{\sigma}(v)$. If $M_{\sigma}(v)$ is birational to the Hilbert scheme $S^{[n]}$, then also the converse implication holds true, i.e. in this case $F_{i} M_{\sigma}(v)=\left\{\mathcal{E} \in M_{\sigma}(v) \mid \operatorname{ch}_{2}(\mathcal{E}) \in S_{i} \mathrm{CH}_{0}(S)\right\}$.

We remark that both parts of the theorem can equally be formulated with $c_{2}$ instead of $\mathrm{ch}_{2}$.

This opens the door to finding infinitely many examples of constant cycle or algebraically coisotropic subvarieties in $M=M_{H}(0,2 H,-1)$. For example, a straightforward application yields.

Lemma 1.3 (Corollary 3.9) The fiber $F=f^{-1}(D)$ over $D \in|2 H|$ is a constant cycle Lagrangian if and only if $D \subset S$ is a constant cycle curve.

Or one can prove, that given $\mathcal{E} \in M$ such that $\operatorname{Supp}(\mathcal{E})=D$, then $\mathcal{E} \in F_{g(\tilde{D})} M$, where $g(\tilde{D})$ is the geometric genus of $D$. Here, the geometric genus of $D$ is the genus of the normalization of $D$ (resp. of $D_{\text {red }}$ ) and the sum over the genera of the normalizations of the irreducible components if $D$ is reducible. This way, we find algebraically coisotropic subvarieties over singular curves. Precisely, for $i=0, \ldots, 4$ let

$$
V_{i}:=\{D \in|2 H| \mid g(\tilde{D}) \leq i\} \subset|2 H|
$$

and set $M_{V_{i}}:=f^{-1}\left(V_{i}\right)$.

Proposition 1.4 (Proposition 4.4) The subvarieties $M_{V_{i}}$ are equidimensional of codimension $n-i$ and satisfy

$$
M_{V_{i}} \subset F_{i} M
$$

In particular, they are algebraically coisotropic.

Actually, $V_{i}$ is reducible due to reducible and non-reduced curves in the linear system $|2 H|$. For every component we find the isotropic fibration and comment on the resulting constant cycle subvarieties. Most of them are rational. However, over the component of non-reduced curves $\Delta \subset V_{2}$, we find three-dimensional constant cycle subvarieties that are not rational (cf. Proposition 4.7).

Another series of examples comes from Brill-Noether theory. Let $B^{\circ} \subset B$ be the locus of smooth curves and $\mathcal{C}^{\circ} \rightarrow B^{\circ}$ the restricted universal curve. For any $i$, we have an isomorphism

$$
M_{H}(0,2 H, i-4)^{\circ} \cong \operatorname{Pic}_{\mathcal{C}^{\circ} / B^{\circ}}^{i},
$$

where $M_{H}(0,2 H, i-4)^{\circ}$ is the preimage of $B^{\circ}$ under the support map $M_{H}(0,2 H, i-4) \rightarrow B$. For $i=1, \ldots 4$, we define

$$
\mathrm{BN}_{i}^{0}\left(B^{\circ}\right):=\left\{\mathcal{L} \in M_{H}(0,2 H, i-4)^{\circ} \mid H^{0}(S, \mathcal{L}) \neq 0\right\} \subset M_{H}(0,2 H, i-4)^{\circ} .
$$


We consider the closures for odd $i$. Namely,

$$
Z_{1}:=\overline{\mathrm{BN}_{1}^{0}\left(B^{\circ}\right)} \subset M_{H}(0,2 H,-3) \text { and } Z_{3}:=\overline{\mathrm{BN}_{3}^{0}\left(B^{\circ}\right)} \subset M:=M_{H}(0,2 H,-1) .
$$

As $M_{H}(0,2 H,-3)$ and $M$ are isomorphic (Lemma 2.1), $Z_{1}$ can also be seen as subvarieties in $M$.

Proposition 1.5 (Proposition 4.1) The subvarieties $Z_{i} \subset M, i=1,3$ have codimension $5-i$ and satisfy

$$
Z_{i} \subset F_{i} M
$$

In particular, they are algebraically coisotropic.

\subsection{Outline}

In Sect. 2, we collect general results on the Mukai system and describe the nature of its fibers. This requires an analysis of the singular curves in $|2 H|$. In Sect. 3, we state Theorems 1.1 and 1.2 in more detail and apply them to $M=M_{H}(0,2 H,-1)$. Section 4 is devoted to present explicit examples. These include the examples from Brill-Noether theory (Sect. 4.1), the examples from singular curves together with their isotropic fibrations (Sect. 4.2) and a less conceptual mixture of examples of constant cycle Lagrangians and examples in $S^{[5]}$ (Sect. 4.3).

\section{The Mukai system}

Let $(S, H)$ be a polarized K3 surface of genus 2 such that the linear system $|H|$ contains a smooth irreducible curve, i.e. $S$ is a double covering $\pi: S \rightarrow \mathbb{P}^{2}$ ramified over a smooth sextic curve $R \subset \mathbb{P}^{2}$ and $H=\pi^{*} \mathcal{O}_{\mathbb{P}^{2}}(1)$ is primitive. We consider the moduli space $M=M_{H}(0,2 H, s)$ of $H$-Gieseker stable coherent sheaves on $S$ with Mukai vector $v=(0,2 H, s)$ where $s \equiv 1 \bmod 2$. This is an irreducible holomorphic symplectic variety of dimension 10 , which is birational to $S^{[5]}$. A point in $M_{H}(0,2 H, s)$ corresponds to a stable sheaf $\mathcal{E}$ on $S$ such that $\mathcal{E}$ is pure of dimension one with support in the linear system $|2 \mathrm{H}|$ and $\chi(\mathcal{E})=s$. Taking the (Fitting) support defines a Lagrangian fibration

$$
f: M_{H}(0,2 H, s) \longrightarrow B:=|2 H| \cong \mathbb{P}^{5}
$$

known as the Mukai system of rank two and genus two [3, 23].

As tensoring with $\mathcal{O}_{S}(H)$ induces an isomorphism

$$
\tau_{H}: M_{H}(0,2 H, s) \stackrel{\sim}{\rightarrow} M_{H}(0,2 H, s+4),
$$

it is immediate that the isomorphism class of $M_{H}(0,2 H, s)$ depends only on $s$ modulo 4 . The following lemma shows that actually the isomorphism class is the same for all odd $s$. If Pic $(S)=\mathbb{Z} \cdot H$ one could also characterize $M_{H}(0,2 H, s)$ for odd $s$ as the unique birational model of $S^{[5]}$ admitting a Lagrangian fibration.

Lemma 2.1 There is an isomorphism 


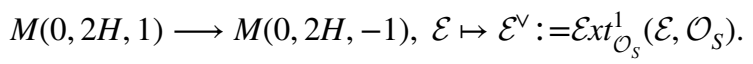

In particular, all the moduli spaces $M_{H}(0,2 H, s)$ for odd s are isomorphic.

Proof Every $\mathcal{E} \in M(0,2 H, 1)$ is pure of dimension one. Therefore, $\mathcal{E} x t_{\mathcal{O}_{S}}^{i}\left(\mathcal{E}, \mathcal{O}_{S}\right)=0$ for $i \neq 1$ and the natural map

$$
\mathcal{E} \stackrel{\sim}{\rightarrow} \mathcal{E}^{\vee \vee}=\mathcal{E} x t_{\mathcal{O}_{S}}^{1}\left(\mathcal{E} x t_{\mathcal{O}_{S}}^{1}\left(\mathcal{E}, \mathcal{O}_{S}\right), \mathcal{O}_{S}\right)
$$

is an isomorphism, [17, Proposition 1.1.10]. Finally, $\mathcal{E}^{\vee}$ is again pure of dimension one and so is any subsheaf $\mathcal{F} \subset \mathcal{E}^{\vee}$. Any subsheaf $\mathcal{F}$ that destabilizes $\mathcal{E}^{\vee}$ would yield a destabilizing quotient of $\mathcal{E} \cong \mathcal{E}^{\vee \vee}$ after dualizing. Hence, $\mathcal{E}^{\vee}$ is again $H$-Gieseker stable.

In the following, we usually choose $s=-1$ and set

$$
M:=M_{H}(0,2 H,-1) \text {. }
$$

With this choice of $s$, a stable vector bundle of rank two and degree one on a smooth curve $C \in|H|$ defines a point in $M$.

\subsection{The linear systems $|H|$ and $|2 H|$}

The geometry of the Mukai system is closely related to the structure of the curves in the linear systems $|H|$ and $|2 H|$, which we want to analyze in this section. A curve in the linear system $|H|$ (resp. $|2 H|$ ) has geometric genus 2 (resp. 5). We use the Segre map $m:|H| \times|H| \rightarrow|2 H|$ to define the subloci

$$
\Delta:=m\left(\Delta_{|H|}\right) \subset \Sigma:=\operatorname{im}(m) \subset|2 H| .
$$

Then $\Sigma \cong \operatorname{Sym}^{2}|H|$ is four-dimensional and its generic member is reduced and has two smooth irreducible components in the linear system $|H|$ meeting transversally in two points. The subset $\Delta \cong|H| \cong \mathbb{P}^{2}$ is the locus of non-reduced curves. If $\rho(S)=1$, then $\Sigma$ is exactly the locus of non-integral curves.

Recall that $\pi: S \rightarrow|H| \cong \mathbb{P}^{2}$ is a double covering, which is ramified along a smooth sextic curve $R \subset \mathbb{P}^{2}$. We have

$$
H^{0}\left(S, \mathcal{O}_{S}(k H)\right) \cong H^{0}\left(\mathbb{P}^{2}, \mathcal{O}_{\mathbb{P}^{2}}(k)\right) \oplus H^{0}\left(\mathbb{P}^{2}, \mathcal{O}_{\mathbb{P}^{2}}(k-3)\right),
$$

and so in particular

$$
H^{0}\left(S, \mathcal{O}_{S}(k H)\right) \cong H^{0}\left(\mathbb{P}^{2}, \mathcal{O}_{\mathbb{P}^{2}}(k)\right) \text { if } k=1,2
$$

We conclude that every curve in $|H|$ (resp. in $|2 H|$ ) is the pullback of a line $\ell$ (resp. a quadric $Q$ ) in $\mathbb{P}^{2}$. In particular, every curve in $|H|$ (resp. in $|2 H|$ ) has singularities depending on the intersection behavior of the ramification sextic $R$ with $\ell$ (resp. $Q$ ) and has at most two (resp. four) irreducible components. For example, let $\ell \subset \mathbb{P}^{2}$ be a line and $C:=\pi^{-1}(\ell) \in|H|$. Assume that $C$ is reducible. Then $C$ consists of two irreducible components $C_{1}$ and $C_{2}$, each isomorphic to $\mathbb{P}^{1}$ with $C_{1} \cdot C_{2}=3$. This is only possible if $\rho(S) \geq 2$. If $\rho(S)=1$, then all curves $C \in|H|$ are irreducible. 
For an ample line bundle $L$ on $S$ and $0 \leq i \leq \frac{L^{2}}{2}+1$, we can consider the closed subvariety

$$
V(i,|L|):=\{D \in|L| \mid g(\tilde{D}) \leq i\} \subset|L|,
$$

which is called a (generalized) Severi variety. We have $\operatorname{dim} V(i,|L|) \leq i$ and there are various results about non-emptiness, irreducibility or smoothness of $V(i,|L|)$ in the literature, e.g. [9]. In our situation, one easily gets a description of $V(i,|H|)$ using the geometry of the covering $\pi: S \rightarrow \mathbb{P}^{2}$.

Proposition 2.2 The varieties $V(i,|H|)$ are non-empty of dimension i for $i=0,1$. Moreover, $V(1,|H|)$ is irreducible and the locus of nodal curves is dense in $V(1,|H|)$. More precisely, any curve in $V(1,|H|) \backslash V(0,|H|)$ is irreducible and has exactly one node or one cusp as singularities. If $(S, H)$ is general, then $V(1,|H|) \subset|H| \cong \mathbb{P}^{2}$ is a curve of degree 30 and $V(0,|H|)$ consists of 324 points. Moreover, any curve in $V(0,|H|)$ has exactly two nodes as singularities.

Proof From the above discussion, we know that $V(1,|H|)$ is parameterized by the tangents of $R$, i.e.

$$
V(1,|H|) \cong R^{\vee} \subset\left(\mathbb{P}^{2}\right)^{\vee}=\left|\mathcal{O}_{\mathbb{P}^{2}}(1)\right|,
$$

and for a smooth sextic $R$ its dual curve $R^{\vee}$ has degree 30 . A curve in $V(1,|H|)$ is nodal if it corresponds to a tangent line that is tangent to $R$ in exactly one point. Hence, this locus is dense. A general smooth sextic has exactly 324 bitangents [14, IV Exercise 2.3].

Next, we study the linear system $|2 H|$ and define

$$
V_{i}:=V(i,|2 H|)
$$

for $i=0, \ldots, 5$. Recall that $m:|H| \times|H| \rightarrow|2 H|$ was the map coming from the Segre embedding. We set

$$
\Sigma_{\{i, j\}}:=m(V(i,|H|) \times V(j,|H|)) \subset V_{i+j}
$$

for $0 \leq i \leq j \leq 2$ and

$$
\Delta_{1}:=m\left(\Delta_{V(1,|H|)}\right) \subset V_{1},
$$

i.e. $\Sigma_{\{i, j\}} \subset \Sigma$ is the locus of reducible curves, whose components have geometric genus bounded by $i$ and $j$, respectively and $\Delta_{1} \subset \Delta$ is the locus of non-reduced curves, with underlying singular curve. We keep writing $\Sigma$ for $\Sigma_{\{2,2\}}$.

Corollary 2.3 We have

$$
\operatorname{dim} \Sigma_{\{i, j\}}=i+j \quad \text { and } \quad \operatorname{dim} \Delta_{1}=1 .
$$

Moreover, $\Sigma_{\{i, j\}}$ for $i \neq 0$ and $\Delta_{1}$ are irreducible.

Finally, we let 


$$
\Lambda_{i}:=\overline{\left\{D \in V_{i} \mid D \text { is integral }\right\}} \subset V_{i},
$$

for $i=0, \ldots, 4$, which turn out to be irreducible for $i \neq 0$.

Lemma 2.4 The varieties $\Lambda_{i}$ are non-empty of dimension $i$ for $i=0, \ldots, 4$. Moreover, if $i \neq 0$, a general curve in $\Lambda_{i}$ has exactly $5-i$ nodes as its only singularities.

Proof We apply the analogous considerations as the for the linear system $|H|$. This is, in order to analyze the structure of $V_{i}$, we study the double coverings $D \rightarrow Q$ of irreducible quadrics $Q \subset \mathbb{P}^{2}$ that arise as pullback of $\pi: S \rightarrow \mathbb{P}^{2}$. Note that any such quadric is isomorphic to $\mathbb{P}^{1}$. Assume that $D$ is reducible, then as above, $D$ is necessarily rational and this is only possible for $\rho(S) \geq 2$. If $D$ is irreducible, its singularities can be read off the intersection of $Q$ with the ramification sextic $R$, yielding the desired result. For example, assume $D$ is integral with $g(\tilde{D})=4$. Then, either $Q \cap R$ consists of 11 points all of which have multiplicity one except one point which has multiplicity two, or $Q \cap R$ consists of 10 points all of which have multiplicity one except one point which has multiplicity three. This produces either one node or one cusp in $D$. The first case provides a codimension one locus in the linear system $\left|\mathcal{O}_{\mathbb{P}^{2}}(2)\right| \cong \mathbb{P}^{5}$ of quadrics in $\mathbb{P}^{2}$, the second case has codimension two.

We sum up our discussion in the following proposition.

Proposition 2.5 The Severi varieties $V_{i} \subset|2 H|, i=0, \ldots, 4$ are non-empty of pure dimension $i$. For $i \neq 0$, their irreducible components correspond to integral, reducible and nonreduced curves, respectively. More precisely, we have

$$
\begin{aligned}
& V_{4}=\Lambda_{4} \cup \Sigma \\
& V_{3}=\Lambda_{3} \cup \Sigma_{\{1,2\}} \\
& V_{2}=\Lambda_{2} \cup \Sigma_{\{0,2\}} \cup \Sigma_{\{1,1\}} \cup \Delta \\
& V_{1}=\Lambda_{1} \cup \Sigma_{\{0,1\}} \cup \Delta_{1} .
\end{aligned}
$$

Here, all varieties occurring on the right hand side but $\Sigma_{\{0,2\}}$ and $\Sigma_{\{0,1\}}$ are irreducible.

\section{Proof}

Note that $V_{4}=\Lambda_{4} \cup \Sigma \subset|2 H| \cong \mathbb{P}^{5}$ is the discriminant divisor of $f$. We compute the degree of its components.

Lemma 2.6 We have

$$
\operatorname{deg}[\Sigma]=3 \text { and } \operatorname{deg}\left[\Lambda_{4}\right]=42 .
$$

In particular, the discriminant divisor off has degree 45.

Proof The easiest way, to see that $\operatorname{deg}[\Sigma]=3$ is a geometric argument. Choose 4 points $x_{1}, \ldots, x_{4}$ in general position and consider the line $\ell=\left\{D \in|2 H| \mid x_{i} \in D\right.$ for all $\left.i=1, \ldots 4\right\}$. There is a unique (resp. no) curve $C \in|H|$ passing through two (resp. three) points in general position. Hence, $\operatorname{deg} \Sigma=\#(\ell \cap \Sigma)=3$, corresponding to the three possible partitions of $x_{1}, \ldots, x_{4}$ into pairs of two points. 
Alternatively, after a choice of coordinates $\Sigma \cong \operatorname{Sym}^{2} \mathbb{P}^{2}$ is embedded into $\mathbb{P}^{5}$ via the map induced by

$$
\begin{aligned}
\mathbb{P}^{2} \times \mathbb{P}^{2} & \rightarrow \mathbb{P}^{5} \\
{\left[x_{0}: x_{1}: x_{2}\right],\left[y_{0}: y_{1}: y_{2}\right] } & \mapsto\left[x_{0} y_{0}: x_{1} y_{1}: x_{2} y_{2}: x_{0} y_{1}+x_{1} y_{0}: x_{0} y_{2}+x_{2} y_{0}: x_{1} y_{2}+x_{2} y_{1}\right] .
\end{aligned}
$$

One checks that the image is cut out by the equation,

$$
f_{0} f_{5}^{2}+f_{1} f_{4}^{2}+f_{2} f_{3}^{2}=4 f_{0} f_{1} f_{2}+f_{3} f_{4} f_{5},
$$

where the coordinates $f_{i}$ of $\mathbb{P}^{5}$ are ordered as in (2.4).

To prove $\operatorname{deg}\left[\Lambda_{4}\right]=45$, we use the computation from $[28, \S 5]$. Let $\mathcal{C}^{\prime}=\bigcup_{t \in \mathbb{P}^{1}} C_{t}$ be a general pencil of curves in the linear system $B=|2 H|$, i.e. $\mathcal{C}^{\prime}=\mathcal{C} \cap\left(S \times \mathbb{P}^{1}\right)$, where $\mathcal{C} \subset S \times B$ is the universal curve and $\mathbb{P}^{1} \subset B$ a general line. Then $\mathcal{C}^{\prime} \subset S \times \mathbb{P}^{1}$ is defined by a section $s \in H^{0}\left(S \times \mathbb{P}^{1}, \mathcal{O}_{S}(2 H) \otimes \mathcal{O}_{\mathbb{P}^{1}}(1)\right)$ and

$$
\mathcal{C}_{\text {sing }}^{\prime}:=V(s \oplus d s)=\bigcup_{t \in \mathbb{P}^{1}}\left(C_{t}\right)_{\text {sing }}
$$

is the union of the singular points of $C_{t}$, where $d s \in H^{0}\left(S \times \mathbb{P}^{1}, \Omega_{S}(2 H) \bigotimes \mathcal{O}_{\mathbb{P}^{1}}(1)\right)$ is the image of $s$ under the natural morphism $d \bigotimes$ id : $\mathcal{O}_{S}(2 H) \bigotimes \mathcal{O}_{\mathbb{P}^{1}}(1) \rightarrow \Omega_{S}(2 H) \bigotimes \mathcal{O}_{\mathbb{P}^{1}}(1)$. We compute

$$
\operatorname{deg} c_{3}\left(\left(\mathcal{O}_{S}(2 H) \bigotimes \mathcal{O}_{\mathbb{P}^{1}}(1)\right) \oplus\left(\Omega_{S}(2 H) \bigotimes \mathcal{O}_{\mathbb{P}^{1}}(1)\right)\right)=48,
$$

i.e. $\mathcal{C}_{\text {sing }}^{\prime}$ consists of 48 points. As a general pencil contains three curves in $\Sigma$ which have two singular points and a generic integral singular curve has exactly one nodal singularity, we conclude $\operatorname{deg} \Lambda_{4}=42$.

Remark 2.7 In [28, §5] the computation (2.5) serves as a demonstration for a formula of the degree of the discriminant locus of a Lagrangian fibration with 'good singular fibers'. An example of such a fibration is the Beauville-Mukai system over a primitive curve class and the discriminant divisor is irreducible of degree $6(n+3)$, where $n$ is the dimension of the base of the fibration. However, in our example the fibers over $\Delta$ are not 'good singular fibers' and we find a different result.

\subsection{Fibers of the Mukai morphism and structure of $M$}

In this section, we collect some information on the fibers of the Mukai morphism.

The moduli space $M=M_{H}(0,2 H,-1)$ contains a dense open subset consisting of the sheaves that are line bundles on their support. The restriction of the Mukai morphism to this locus is smooth [20, Proposition 2.8] and the image of the restricted morphism is $B \backslash \Delta$ [8, Lemma 3.5.3]. In particular, $M_{\Sigma}:=f^{-1}(\Sigma)$ contains a dense open subset that parameterizes the push forwards of line bundles, but $M_{\Delta}:=f^{-1}(\Delta)$ does not.

Following [8, Propositions 3.7.1 and 3.7.23], we can give a description of the fibers of the Mukai morphism. To this end, first assume that $\operatorname{Pic}(S)=\mathbb{Z} \cdot H$. The fibers of the Mukai morphism $f: M \rightarrow B$ have the following properties: 


$$
f^{-1}(x)= \begin{cases}\text { is reduced and irreducible } & \text { if } x \in B \backslash \Sigma \\ \text { is reduced and has two irreducible components } & \text { if } x \in \Sigma \backslash \Delta \\ \text { has two irreducible components with multiplicities } & \text { if } x \in \Delta .\end{cases}
$$

Let us make this more precise for generic points:

- In the first case, let $x \in B \backslash \Sigma$ correspond to a smooth curve $D$, then $f^{-1}(x) \cong \operatorname{Pic}^{3}(D)$.

- In the second case, let $x \in \Sigma \backslash \Delta$ correspond to the union $D=D_{1} \cup D_{2}$ of two smooth curves meeting transversally in two points. Then $f^{-1}(x)$ contains a dense open subset parameterizing line bundles on $D$. Following [8, Lemma 3.3.2], the two irreducible components of $f^{-1}(x)$ correspond to line bundles with partial degree $(2,1)$ and $(1,2)$.

- In the third case, let $x \in \Delta$ correspond to a non-reduced curve with smooth underlying curve $C \in|H|$. Then $f^{-1}(x)$ has two non-reduced irreducible components, which we denote as follows

$$
M_{2 C}:=f^{-1}(x)_{\text {red }}=M_{2 C}^{0} \cup M_{2 C}^{1} .
$$

The first component $M_{2 C}^{0}$ consists of those sheaves, that are pushed forward from the reduced curve $C$. With its reduced structure it is isomorphic to the moduli space of stable vector bundles of rank two and degree one on $C$. The other component $M_{2 C}^{1}$ is the closure of those sheaves that can not be endowed with an $\mathcal{O}_{C}$-module structure. All these sheaves fit into a short exact sequence

$$
0 \rightarrow i_{*}\left(\mathcal{L}(x) \otimes \omega_{C}^{-1}\right) \rightarrow \mathcal{E} \rightarrow i_{*} \mathcal{L} \rightarrow 0,
$$

where $i: C \hookrightarrow S$ is the inclusion, and $\mathcal{L} \in \operatorname{Pic}^{1}(C)$ is the torsionfree part of $\left.\mathcal{E}\right|_{C}$ and $x \in C$ is the support of the torsion part of $\left.\mathcal{E}\right|_{C}$. This extension is intrinsically associated to $\mathcal{E}$, for details see [15]. Following, [8, Propositions 3.7.19 \& 3.7.23], the decomposition (2.7) remains valid if the underlying curve $C$ is irreducible with nodal or cuspidal singularities. (In particular, in the case that $\rho(S)=1$, it holds for every $x \in \Delta$.)

In the case of a K3 surface of higher Picard rank, the general picture remains the same. But due to reducible curves in the linear system $|H|$ or $B \backslash \Sigma$, the fibers could exceptionally have more irreducible components. For example, if $x \in B \backslash \Sigma$ corresponds to a reducible curve with two smooth components, then $f^{-1}(x)$ still contains a dense open subset parameterizing line bundles. However, following de Cataldo et al. [8, Lemma 3.3.2] one finds, that the numerical restrictions imposed by the stability now allow partial degrees $(5,-1),(4,0),(3,1),(2,2),(1,3),(0,4),(-1,5)$. Thus, in this case $f^{-1}(x)$ has seven irreducible components.

The decomposition (2.7) also exists globally over the locus of curves $D=2 C$ with $C \in|H|$ smooth, which we denote by $\Delta^{\circ} \subset \Delta$. Here, we have

$$
M_{\Delta^{\circ}}=f^{-1}\left(\Delta^{\circ}\right)_{\text {red }}=M_{\Delta^{\circ}}^{0} \cup M_{\Delta^{\circ}}^{1},
$$

where $M_{\Delta^{\circ}}^{0}$ is a relative moduli space of stable vector bundles and $M_{\Delta^{\circ}}^{1}$ the closure of its complement, [8, Proposition 3.7.23]. We set

$$
M_{\Delta}^{0}=\overline{M_{\Delta^{\circ}}^{0}} \text { and } M_{\Delta}^{1}=\overline{M_{\Delta^{\circ}}^{1}} .
$$




\section{Orbits under rational equivalence}

Our strategy to find algebraically coisotropic subvarieties is to single out points whose orbit under rational equivalence has a high dimension. In this section, we explain how this can be done combining results of Voisin [31, 32], Shen-Yin-Zhao [29] and Marian-Zhao [22] (for the precise references, see below).

\subsection{Preliminaries}

We start by recalling some general definitions. Let $(X, \sigma)$ be an irreducible holomorphic symplectic manifold of dimension $2 n$. For a smooth subvariety $Y \subset X$, we let

$$
\mathcal{T}_{Y}^{\perp}:=\operatorname{ker}\left(\mathcal{T}_{X} \tilde{\rightarrow} \Omega_{X} \rightarrow \Omega_{Y}\right),
$$

where the first arrow is given by $\sigma$.

(i) A subvariety $Y \subset X$ is a constant cycle subvariety [18] if all its points are rationally equivalent in $X$. Note that this is the case, if $Y$ contains a dense open subset $U$, such that all points in $U$ are rationally equivalent in $X$. Mumford's theorem [24] implies that a constant cycle subvariety $Y \subset X$ is isotropic [32, Corollary 1.2], i.e.

$$
\mathcal{T}_{Y_{\text {reg }}} \subset \mathcal{T}_{Y_{\text {reg }}}^{\perp} \text { or equivalently }\left.\sigma\right|_{Y_{\text {reg }}}=0 \text {, }
$$

where $Y_{\text {reg }}$ denotes the regular part of $Y$. In particular, $\operatorname{dim} Y \leq n$ and if $\operatorname{dim} Y=n$, then $Y$ is a Lagrangian subvariety.

(ii) A subvariety $Z \subset X$ is algebraically coisotropic [32, Def. 0.6] if $Z$ is coisotropic (i.e. $\mathcal{T}_{Z_{\text {reg }}}^{\perp} \subset \mathcal{T}_{Z_{\text {reg }}}$ ) and the corresponding foliation is algebraically integrable. For a subvariety of codimension $i$, this is equivalent to the existence of a $2 n-2 i$-dimensional variety $T$ and a rational surjective map $\phi: Z \rightarrow T$ such that

$$
\mathcal{T}_{Z_{\text {reg }}^{1}}=\operatorname{ker}\left(\mathcal{T}_{Z^{\circ}} \rightarrow \phi^{*} \mathcal{T}_{T}\right) \text { on } Z^{\circ} \cap Z_{\text {reg }},
$$

where $Z^{\circ} \subset Z$ is the open subset, where $\phi$ is defined and smooth, or equivalently

$$
\left.\sigma\right|_{Z}=\phi^{*} \sigma_{T} \text { for some }(2,0) \text { form } \sigma_{T} \text { on } T \text {. }
$$

Actually, $T$ and $\phi$ are unique up to birational equivalence. We call $\phi$ the associated isotropic fibration.

(iii) For a point $x \in X$, its orbit under rational equivalence is

$$
O_{x}:=\left\{x^{\prime} \in X \mid[x]=\left[x^{\prime}\right] \in \mathrm{CH}_{0}(X)\right\} \subset X,
$$

which is a countable union of closed algebraic subvarieties [30, Lemma 10.7]. Its dimension is defined to be the supremum over the dimensions of its irreducible components.

Following Voisin ${ }^{1}$ [32, Def 0.2], we set

\footnotetext{
1 We reversed the numbering from Voisin [32].
} 


$$
F_{i} X:=\left\{x \in X \mid \operatorname{dim} O_{x} \geq n-i\right\} .
$$

for $i=0, \ldots, n$. This is again a countable union of closed algebraic subvarieties and defines a filtration on the points of $X$

$$
F_{0} X \subset F_{1} X \subset \cdots \subset F_{n} X=X .
$$

From [32, Theorem 1.3] it is known that

$$
\operatorname{dim} F_{i} X \leq n+i
$$

and conjecturally [32, Conj 0.4 ] equality holds true. The following theorem says that a component of maximal dimension is algebraically coisotropic.

Theorem 3.1 [32, Theorem 0.7] Let $Z \subset X$ be a subvariety of codimension $n-i$ such that $Z \subset F_{i} X$, then $Z$ is algebraically coisotropic and the fibers of the associated isotropic fibration $\phi: Z \rightarrow T$ are constant cycle subvarieties of dimension $n-i$.

Now, let $X=M_{\sigma}(v)$ be a smooth, projective moduli space of (Bridgeland-)stable objects in $D^{b}(S)$. In this situation, we have the following beautiful criterion for rational equivalence.

Theorem 3.2 [22, Theorem], [29, Conj 0.3] Two points $\mathcal{E}, \mathcal{E}^{\prime} \in M_{\sigma}(v)$ satisfy

$$
[\mathcal{E}]=\left[\mathcal{E}^{\prime}\right] \in \mathrm{CH}_{0}\left(M_{\sigma}(v)\right)
$$

if and only if

$$
\operatorname{ch}_{2}(\mathcal{E})=\operatorname{ch}_{2}\left(\mathcal{E}^{\prime}\right) \in \mathrm{CH}_{0}(S) .
$$

Remark 3.3 $\mathrm{As} \mathrm{ch}_{2}(\mathcal{E})=\frac{1}{2} c_{1}(\mathcal{E})^{2}-c_{2}(\mathcal{E})$, and $c_{1}(\mathcal{E})$ is fixed for all $\mathcal{E} \in M_{\sigma}(v)$, one could also phrase the theorem using $c_{2}$.

In particular, for $\mathcal{E} \in M_{\sigma}(v)$ we have

$$
O_{\mathcal{E}}=\left\{\mathcal{E}^{\prime} \in M_{\sigma}(v) \mid \operatorname{ch}_{2}\left(\mathcal{E}^{\prime}\right)=\operatorname{ch}_{2}(\mathcal{E}) \in \mathrm{CH}_{0}(S)\right\} \subset M_{\sigma}(v) .
$$

Using that the union of all constant cycle curves in $S$ is Zariski dense and Theorem 3.2 allow one to prove the following theorem.

Theorem $3.4[29$, Theorem $0.5(i)]$ For all $0 \leq i \leq n$ there is an algebraically coisotropic subvariety $Z \subset M_{\sigma}(v)$ of codimension $i$ such that the isotropic fibration $Z \rightarrow T$ has generically constant cycle fibers of dimension $i$. In particular,

$$
\operatorname{dim} F_{i} M_{\sigma}(v)=n+i
$$

i.e. (3.2) is actually an equality.

Next, one could ask how the filtration $F_{i} M_{\sigma}(v)$ interferes with the second Chern classes. The answer is to consider O'Grady's filtration on $\mathrm{CH}_{0}(S)$. Let us recall some results about $\mathrm{CH}_{0}(S)$. 
In [4], Beauville and Voisin prove that any point lying on a rational curve in $S$ determines the same class

$$
c_{S} \in \mathrm{CH}_{0}(S),
$$

which has the property that the image of the intersection product $\operatorname{Pic}(S) \otimes \operatorname{Pic}(S) \rightarrow \mathrm{CH}_{0}(S)$ is contained in $\mathbb{Z} \cdot c_{S}$. In [27], building on this class, now called the Beauville-Voisin class, O'Grady introduces an increasing filtration $S$. on $\mathrm{CH}_{0}(S)$,

$$
S_{0} \mathrm{CH}_{0}(S) \subset S_{1} \mathrm{CH}_{0}(S) \subset \ldots \subset S_{i} \mathrm{CH}_{0}(S) \subset \ldots \subset \mathrm{CH}_{0}(S),
$$

where $S_{i} \mathrm{CH}_{0}(S)$ is the union of cycles of the form $[z]+d \cdot c_{S}$ for some effective zero-cycle $z$ of degree $i$ and $d \in \mathbb{Z}$. In particular, $S_{0} \mathrm{CH}_{0}(S)=\mathbb{Z} \cdot c_{S}$. O'Grady's filtration $S_{.} \mathrm{CH}_{0}(S)$ has several useful properties, [27, Corollary. 1.7 and Claim 0.2]:

(1) The filtration is compatible with addition, i.e. if $\alpha \in S_{i} \mathrm{CH}_{0}(S)$ and $\beta \in S_{j} \mathrm{CH}_{0}(S)$, then $\alpha+\beta \in S_{i+j} \mathrm{CH}_{0}(S)$.

(2) Each step of the filtration $S_{i} \mathrm{CH}_{0}(S)$ is closed under multiplication with $\mathbb{Z}$, i.e. if $\alpha \in S_{i} \mathrm{CH}_{0}(S)$ then $m \cdot \alpha \in S_{i} \mathrm{CH}_{0}(S)$ for every $m \in \mathbb{Z}$.

(3) If $C$ is an irreducible, smooth projective curve and $f: C \rightarrow S$ is a non-constant morphism, then

$$
f_{*} \mathrm{CH}_{0}(C) \subset S_{g(C)} \mathrm{CH}_{0}(S) .
$$

Theorem 3.5 [29, Theorem 0.5(ii)] Let $M_{\sigma}(v)$ be a smooth projective moduli space of Bridgeland stable objects in $D^{b}(S)$ with $\operatorname{dim} M_{\sigma}(v)=2 n$. If $\mathcal{E} \in M_{\sigma}(v)$ satisfies $\mathrm{ch}_{2}(\mathcal{E}) \in S_{i} \mathrm{CH}_{0}(S)$, then $\mathcal{E} \in F_{i} M_{\sigma}(v)$. Moreover, if $M_{\sigma}(v)$ is birational to the Hilbert scheme $S^{[n]}$, then also the converse implication holds true, i.e. in this case

$$
F_{i} M_{\sigma}(v)=\left\{\mathcal{E} \in M_{\sigma}(v) \mid \operatorname{ch}_{2}(\mathcal{E}) \in S_{i} \mathrm{CH}_{0}(S)\right\} .
$$

Note that $c_{1}(\mathcal{E})^{2} \in \mathrm{CH}_{0}(S)$. Hence, the theorem can equally be the formulated using $c_{2}$ instead of $\mathrm{ch}_{2}$.

Proof We sketch the proof along the lines of Shen et al. [29, Proof of Theorem 0.5(ii)], where the first part of the theorem is proven. The case of $S^{[n]}$, i.e. that for all $\xi \in S^{[n]}$

$$
\operatorname{dim} O_{\xi} \geq n-i \Longleftrightarrow[\operatorname{Supp}(\xi)] \in S_{i} \mathrm{CH}_{0}(S)
$$

is proven in [31, Theorem 1.4]. Note that only the implication from left to right needs a proof. The other implication follows because any point representing the Beauville-Voisin lies on a rational curve and hence if $\operatorname{Supp}(\xi)$ contains $(n-i) \cdot c_{S}$, we have $\operatorname{dim} O_{\xi} \geq n-i$.

For the general case, let $\mathcal{E} \in M=M_{\sigma}(v)$. By [29, Theorem 0.1], we can write

$$
\operatorname{ch}_{2}(\mathcal{E})=[\operatorname{Supp}(\xi)]+d \cdot\left[c_{S}\right] \in \mathrm{CH}_{0}(S)
$$

for some $\xi \in S^{[n]}$ and $d \in \mathbb{Z}$ depending on the degree of $\operatorname{ch}_{2}(\mathcal{E})$, which is fixed. After knowing the result for $S^{[n]}$, the theorem translates into the statement

$$
\left.\operatorname{dim} O_{\xi} \geq n-i \Rightarrow \operatorname{dim} O_{\mathcal{E}} \geq n-i \text { (resp. } \operatorname{dim} O_{\xi} \geq n-i \Leftrightarrow \operatorname{dim} O_{\mathcal{E}} \geq n-i, \text { if } M \sim_{\text {bir }} S^{[n]}\right) .
$$


The two orbits can be compared by means of the incidence variety

$$
R=\left\{(\mathcal{E}, \xi) \in M \times S^{[n]} \mid \operatorname{ch}_{2}(\mathcal{E})=[\operatorname{Supp}(\xi)]+d \cdot\left[c_{S}\right] \in \mathrm{CH}_{0}(S)\right\},
$$

which is a countable union of Zariski closed subsets in $M \times S^{[n]}$. There exists an irreducible component $R_{0} \subset R$ which projects generically finite and surjective to both factors, and hence yields a correspondence between the two orbits (see [29, §2.3]). However, in order to compare their dimensions, one needs to know that the components of maximal dimension in every orbit under rational equivalence are dense. This is known for the Hilbert scheme, whence the inclusion $S_{i}^{\mathrm{SYZ}} \mathrm{CH}_{0}(M) \subset S_{i}^{\mathrm{V}} \mathrm{CH}_{0}(M)$ always holds. The reverse inclusion is true if $M$ is birational to $S^{[n]}$ but in general not known.

\subsection{Orbits under rational equivalence in $M$}

We turn back to our favorite example $M=M_{H}(0,2 H,-1)$ with the goal in mind, to give explicit constructions of constant cycle subvarieties in $M$. We remark that any moduli space of Gieseker-stable sheaves on a K3 surface $S$ (with respect to a generic polarization) can be realized as a moduli space of Bridgeland-stable objects in $D^{b}(S)$ [7, Proposition 14.2]. In particular, the above results can be applied to $M$.

The first step is to understand the orbits under rational equivalence in $M$ and the filtration

$$
F_{0} M \subset F_{1} M \subset \ldots \subset F_{5} M=M .
$$

Recall that $M$ is birational to $S^{[5]}$ and thus by Theorem 3.5, we know

$$
F_{i} M=\left\{\mathcal{E} \in M \mid \operatorname{dim} O_{\mathcal{E}} \geq 5-i\right\}=\left\{\mathcal{E} \in M \mid \operatorname{ch}_{2}(\mathcal{E}) \in S_{i} \mathrm{CH}_{0}(S)\right\}
$$

and

$$
\operatorname{dim} F_{i} M=5+i
$$

for $0 \leq i \leq 5$. The following lemma is a straightforward computation using the Grothendieck-Riemann-Roch theorem.

Lemma 3.6 Let $i: D \hookrightarrow S$ be a reduced curve and let $\mathcal{F}$ be a vector bundle on $D$.

(i) Assume that $D$ is irreducible and let $v: \tilde{D} \rightarrow D$ be its normalization. Then

$$
\operatorname{ch}_{2}\left(i_{*} \mathcal{F}\right)=i_{*} v_{*} c_{1}\left(v^{*} \mathcal{F}\right)-\operatorname{rk}(\mathcal{F})\left(\frac{1}{2} i_{*} \nu_{*} c_{1}\left(\omega_{\tilde{D}}\right)-\sum_{p \in D} m_{p}[p]\right) \in \mathrm{CH}_{0}(S)
$$

where $m_{p}=$ length $\left(\nu_{*} \mathcal{O}_{\tilde{D}} / \mathcal{O}_{D}\right)_{p}$. In particular,

$$
\operatorname{ch}_{2}\left(i_{*} \mathcal{F}\right) \in \operatorname{im}\left(\mathrm{CH}_{0}(\tilde{D}) \stackrel{i_{*} \nu_{*}}{\longrightarrow} \mathrm{CH}_{0}(S)\right) \subset S_{g(\tilde{D})} \mathrm{CH}_{0}(S) .
$$

(ii) Assume that $D=D_{1} \cup D_{2}$ has two irreducible components. Then

$$
\operatorname{ch}_{2}\left(i_{*} \mathcal{F}\right)=\operatorname{ch}_{2}\left(\left.i_{1 *} \mathcal{F}\right|_{D_{1}}\right)+\operatorname{ch}_{2}\left(\left.i_{2 *} \mathcal{F}\right|_{D_{2}}\right)-\operatorname{rk}(\mathcal{F})\left(D_{1} . D_{2}\right) c_{S} \in \mathrm{CH}_{0}(S)
$$


were $i_{k}: D_{k} \hookrightarrow S, k=1,2$ are the inclusions of the components. In particular,

$$
\operatorname{ch}_{2}\left(i_{*} \mathcal{F}\right) \in S_{g\left(\tilde{D_{1}}\right)+g\left(\tilde{D_{2}}\right)} \mathrm{CH}_{0}(S) \text {. }
$$

Example 3.7 Using Lemma 3.6 we compute $\mathrm{ch}_{2}(\mathcal{E})$ for some cases of stable sheaves $\mathcal{E}$ occuring in $M$ :

(i) Let $\mathcal{E} \in M$ such that $D=\operatorname{Supp}(\mathcal{E})$ is smooth, then $\mathcal{E}=i_{*} \mathcal{L}$, where $i: D \hookrightarrow S$ is the inclusion and $\mathcal{L} \in \operatorname{Pic}^{3}(D)$. We find

$$
\operatorname{ch}_{2}(\mathcal{E})=-4 c_{S}+i_{*} c_{1}(\mathcal{L})
$$

(ii) Let $\mathcal{E} \in M$ be the pushforward of a line bundle $\mathcal{L}$ on its support $D=\operatorname{Supp} \mathcal{E}$ and assume that $D=D_{1} \cup D_{2}$ has two smooth and connected components. We write $\mathcal{E}=i_{*} \mathcal{L}$, then

$$
\operatorname{ch}_{2}(\mathcal{E})=-4 c_{S}+i_{1 *} c_{1}\left(\left.\mathcal{L}\right|_{D_{1}}\right)+i_{2 *} c_{1}\left(\left.\mathcal{L}\right|_{D_{2}}\right)
$$

where $i_{k}: D_{k} \hookrightarrow C, k=1,2$ are the inclusions.

(iii) Let $\mathcal{E} \in M_{2 C}^{0}$ for a smooth curve $C \in|H|$, i.e. $\operatorname{Supp}(\mathcal{E})=2 C$ and $\mathcal{E}=i_{*} \mathcal{E}_{0}$, where $i: C \hookrightarrow S$ is the inclusion and $\mathcal{E}_{0}$ is a vector bundle of rank 2 and degree 1 on $C$. Then

$$
\operatorname{ch}_{2}(\mathcal{E})=-2 c_{S}+i_{*} c_{1}\left(\mathcal{E}_{0}\right) .
$$

(iv) Let $\mathcal{E} \in M_{2 C}^{1} \backslash M_{2 C}^{0}$ for a smooth curve $C \in|H|$, i.e. $\operatorname{Supp}(\mathcal{E})=2 C$ but $\mathcal{E}$ is not pushed forward along the inclusion $i: C \hookrightarrow S$. However, $\mathcal{E}$ fits into a short exact sequence

$$
0 \rightarrow i_{*}\left(\mathcal{L}(x) \otimes \omega_{C}^{-1}\right) \rightarrow \mathcal{E} \rightarrow i_{*} \mathcal{L} \rightarrow 0,
$$

for some $\mathcal{L} \in \operatorname{Pic}^{1}(C)$ and $x \in C$. Hence

$$
\operatorname{ch}_{2}(\mathcal{E})=\operatorname{ch}_{2}\left(i_{*}\left(\mathcal{L}(x) \otimes \omega_{C}^{-1}\right)\right)+\operatorname{ch}_{2}\left(i_{*} \mathcal{L}\right)=-4 c_{S}+[i(x)]+2 i_{*} c_{1}(\mathcal{L})
$$

Corollary 3.8 Let $\mathcal{E} \in M$ and let $D=\operatorname{Supp}(\mathcal{E})$. Assume either that $D$ is reduced and $\mathcal{E}$ is locally free on $D$, or $D$ is non-reduced and $D_{\text {red }}$ is a smooth curve. Then

$$
\operatorname{ch}_{2}(\mathcal{E}) \in F_{g(\tilde{D})} M,
$$

where $g(\tilde{D})$ is the geometric genus of $D$.

As before, the geometric genus of $D$ is the genus the normalization of $D$ (resp. of $D_{\text {red }}$ ) and the sum over the genera of the normalizations of the irreducible components if $D$ is reducible.

Corollary 3.9 Assume that $\rho(S)=1$. The fiber $F=f^{-1}(D)$ over a curve $D \in|2 H|$ is a constant cycle Lagrangian if and only if $D$ is a constant cycle curve in $S$. If $D \in \Delta$ this means that the underlying reduced curve is constant cycle.

Proof It suffices to consider a dense open subset of $F$, in order to decide whether $F$ is a constant cycle subvariety. First assume that $i: D \hookrightarrow S$ is reduced. Then $F$ contains a dense 
open subset parameterizing line bundles of fixed degree. In Lemma 3.6 we saw that the class of $i_{*} \mathcal{L}$ in $\mathrm{CH}_{0}(M)$ depends on

$$
\operatorname{Pic}^{k}(D) \rightarrow \mathrm{CH}_{0}(S), \mathcal{L} \mapsto i_{*} c_{1}(\mathcal{L}),
$$

which is constant if $D$ is a constant cycle curve. Conversely, assume that $F \subset M$ is a constant cycle subvariety. Then in particular,

$$
i_{*} c_{1}\left(\mathcal{O}_{D}(k x)\right)=k i_{*}[x] \in S_{0} \mathrm{CH}_{0}(S)
$$

and hence $[x]=c_{S}$ for all $x \in D$. (We use that $\mathrm{CH}_{0}(S)$ is torsionfree).

If $D=2 C$ is non-reduced, we apply the same argument to the explicit description (2.7) of the fiber $F$. It is exclusively here, that we use $\rho(S)=1$.

Remark 3.10 We expect that a case by case analysis also proves Corollary 3.9 when $\rho(S)>1$. In this case it is no longer true, that all curves $C \in|H|$ are irreducible with at worst nodal or cuspidal singularities.

\section{Algebraically coisotropic subvarieties in $M$}

We give several examples of algebraically coisotropic subvarieties in $M=M_{H}(0,2 H,-1)$.

\subsection{Horizontal examples from Brill-Noether loci}

Brill-Noether theory allows one to produce examples of constant cycle subvarieties.

Let $B^{\circ} \subset B$ be the locus of smooth curves and $\mathcal{C}^{\circ} \rightarrow B^{\circ}$ the restricted universal curve. For any $k$, we have an isomorphism

$$
M_{H}(0,2 H, k-4)^{\circ} \cong \operatorname{Pic}_{\mathcal{C}^{\circ} / B^{\circ}}^{k},
$$

where $M_{H}(0,2 H, k-4)^{\circ}$ is the preimage of $B^{\circ}$ under the support map $M_{H}(0,2 H, k-4) \rightarrow B$. For $k=1,3$, we define

$$
\mathrm{BN}_{k}^{0}\left(B^{\circ}\right):=\left\{\mathcal{L} \in M_{H}(0,2 H, k-4)^{\circ} \mid H^{0}(S, \mathcal{L}) \neq 0\right\} \subset M_{H}(0,2 H, k-4)^{\circ}
$$

and consider the closures

$$
Z_{1}:=\overline{\mathrm{BN}_{1}^{0}\left(B^{\circ}\right)} \subset M_{H}(0,2 H,-3) \text { and } Z_{3}:=\overline{\mathrm{BN}_{3}^{0}\left(B^{\circ}\right)} \subset M .
$$

One can show that $Z_{3}$ is strictly contained in $\mathrm{BN}^{0}(M):=\left\{\mathcal{E} \in M \mid H^{0}(S, \mathcal{E}) \neq 0\right\}$ as $\mathrm{BN}^{0}(M)$ has an additional component over $\Delta$.

Proposition 4.1 The subvarieties $Z_{i} \subset M_{H}(0,2 H, i-4)$ have codimension $5-i$ for $i=1,3$ and satisfy

$$
Z_{i} \subset F_{i} M_{H}(0,2 H, i-4) .
$$

In particular, they are algebraically coisotropic. 
Proof A point in $\mathrm{BN}_{i}^{0}\left(B^{\circ}\right)$ is of the form $\mathcal{E}=i_{*} \mathcal{O}_{D}(\xi)$, where $D \in B^{\circ}$ and $\xi \subset D$ is an effective divisor of degree $i$. Hence

$$
\operatorname{ch}_{2}(\mathcal{E}) \equiv[\operatorname{Supp}(\xi)] \bmod \mathbb{Z} \cdot c_{S}
$$

in $\mathrm{CH}_{0}(S)$ and we conclude $\mathrm{ch}_{2}(\mathcal{E}) \in S_{i} \mathrm{CH}_{0}(S)$, which in turn gives $Z_{i} \subset F_{i} M_{H}(0,2 H, i-4)$. By Theorem 3.2, this implies $\operatorname{dim} Z_{i} \leq 5+i$, whereas the reverse inequality is known from Brill-Noether theory [1, IV Lemma 3.3].

Actually, $Z_{1}$ is a projective bundle over $S$. Precisely, let $D \in|2 H|$ and $\mathcal{L} \in Z_{1} \cap f^{-1}(D)$, i.e. $\mathcal{L} \in \operatorname{Pic}^{1}(D)$ is effective and can uniquely be written as $\mathcal{O}_{D}(x)$ for some $x \in D$. This way, $Z_{1}$ is isomorphic the universal curve $\mathcal{C} \subset|2 H| \times S$, which is a $\mathbb{P}^{4}$ bundle with respect to the second projection. With the same arguments, we also prove that $Z_{3}$ is generically a $\mathbb{P}^{2}$-bundle over $S^{[3]}$, which parameterizes the line bundles $\mathcal{O}_{D}(\xi)$ over $\xi \in S^{[3]}$.

In the following, we will consider $Z_{1}$ as a subvariety of $M$ via the isomorphism

$$
M_{H}(0,2 H,-3) \rightarrow M, \mathcal{E} \mapsto \mathcal{E} x t^{1}\left(\mathcal{E}, \mathcal{O}_{S}\right) \otimes \mathcal{O}_{S}(-H)
$$

In particular, over a smooth curve $D \in|2 H|$, we have

$$
\operatorname{Pic}^{1}(D) \rightarrow \operatorname{Pic}^{3}(D),\left.\mathcal{L} \mapsto \mathcal{L}^{\vee} \otimes \mathcal{O}_{S}(H)\right|_{D}
$$

Lemma 4.2 We have

$$
Z_{1} \subset\left\{\mathcal{E} \in M \mid h^{0}(\mathcal{E}) \geq 2\right\} .
$$

In particular, there is an inclusion

$$
Z_{1} \subset Z_{3}
$$

Proof It suffices to show the result over a smooth curve $D \in|2 H|$. Let $\mathcal{L} \in \operatorname{Pic}^{1}(D)$ such that $H^{0}(D, \mathcal{L}) \neq 0$. We want to show that $\operatorname{dim} H^{0}\left(D,\left.\mathcal{L}^{\vee} \otimes \mathcal{O}_{S}(H)\right|_{D}\right) \geq 2$. Write $\mathcal{L}=\mathcal{O}_{D}(x)$ for a point $x \in D$. On $S$, we have a short exact sequence

$$
\left.0 \rightarrow \mathcal{O}_{S}(-H) \rightarrow \mathcal{I}_{x}(H) \rightarrow \mathcal{O}_{D}(-x) \otimes \mathcal{O}_{S}(H)\right|_{D} \rightarrow 0
$$

and the resulting long exact cohomology sequence proves the lemma.

Remark 4.3 One can also define $Z_{1}$ directly as a subvariety of $M$. Namely, $Z_{1}$ is the closure of the Brill-Noether locus

$$
\mathrm{BN}_{3}^{1}\left(B^{\circ}\right):=\left\{\mathcal{L} \in M^{\circ} \mid h^{0}(S, \mathcal{L}) \geq 2\right\} \subset M,
$$

see [16]. Due to the non-primitivity of the linear system $|2 H|$, unexpected things happen here. Namely, the smooth curves $D \in|2 H|$ are hyperelliptic and we have $W_{3}^{1}(D) \neq \emptyset$ for all irreducible curves $D \in|2 H|$, even though the Brill-Noether number $\rho(5,1,3)=5-2(5-3+1)$ is negative. 


\subsection{Vertical examples from singular curves}

In this section, we give examples of algebraically coisotropic subvarieties, that arise as preimages of subvarieties in $B$. In Corollary 3.9, we already treated the case of a fiber over a point $D \in B$. Namely, $f^{-1}(D)$ is a constant cycle Lagrangian, if and only if $D$ is a constant cycle curve.

We set

$$
M_{V_{i}}:=f^{-1}\left(V_{i}\right)
$$

where $V_{i}:=\{D \in|2 H| \mid g(\tilde{D}) \leq i\}$ for $i=1, \ldots, 4$ was defined in (2.3).

Proposition 4.4 The subvarieties $M_{V_{i}}$ are equidimensional of codimension $5-i$ for $i=1, \ldots, 4$ and satisfy

$$
M_{V_{i}}:=f^{-1}\left(V_{i}\right) \subset F_{i} M .
$$

In particular, they are algebraically coisotropic.

Proof We saw in Proposition 2.5 that $\operatorname{dim} V_{i}=i$ and in Corollary 3.8 that $g(\tilde{D}) \leq i$ implies that $f^{-1}(D) \subset F_{i} M$ for every $D \in|2 H|$.

In the following section, we find the isotropic fibrations for $M_{V_{i}}$

\subsubsection{Isotropic fibrations}

In order to understand the constant cycle subvarieties resulting from the above examples, we write down the isotropic fibration for $M_{\Sigma} \subset M_{V_{4}}$ and $M_{\Delta} \subset M_{V_{2}}$ and $M_{\Lambda_{i}} \subset M_{V_{i}}$ for $i=1, \ldots, 4$.

Proposition 4.5 For every $i=1, \ldots, 4$, there is a quasi-projective scheme $T_{i}$ of dimension $2 i$ fitting into a diagram

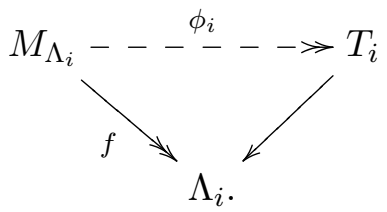

The fibers of $\phi_{i}$ are rational constant cycle subvarieties of $M$ of dimension $5-i$.

Proof A general point in $M_{\Lambda_{i}}$ is the pushforward of a line bundle on a singular curve in $\Lambda_{i}$. Its class in $\mathrm{CH}_{0}(M)$ however, depends only on the pullback of the line bundle to the normalization (cf. Theorem 3.2 and Lemma 3.6). This is what $M_{\Lambda_{i}} \rightarrow T_{i}$ encodes.

Consider the universal curve over $|2 H|$ and let $\mathcal{C}_{i} \rightarrow \Lambda_{i}$ be its restriction to $\Lambda_{i} \subset|2 H|$. By construction, the generic fiber of $\mathcal{C}_{i}$ is singular and so must be the total space $\mathcal{C}_{i}$. Hence, the normalization 


$$
\tilde{\mathcal{C}}_{i} \rightarrow \mathcal{C}_{i}
$$

generically parameterizes the normalization of the curves in $\Lambda_{i}$. We set

$$
T_{i}:=\operatorname{Pic}_{\tilde{C}_{i} / U_{i}}^{3},
$$

where $U_{i} \subset \Lambda_{i}$ is the dense open subset such that $\tilde{\mathcal{C}}_{i}$ has smooth fibers over $U_{i}$. Then pulling back along $\tilde{\mathcal{C}}_{i} \rightarrow \mathcal{C}_{i}$ defines

$$
\phi_{i}: M_{\Lambda_{i}} \supset \operatorname{Pic}_{\mathcal{C}_{i} / \Lambda_{i}}^{3} \rightarrow T_{i}:=\operatorname{Pic}_{\tilde{\mathcal{C}}_{i} / U_{i}}^{3}
$$

and by Lemma 3.6(i) the fibers are constant cycle subvarieties of $M$. Over the open dense subset of curves in $\Lambda_{i}$, that have exactly $5-i$ nodes as their only singularities, the fibers of $\phi_{i}$ are isomorphic to $\mathbb{G}_{m}^{5-i}$.

Proposition 4.6 There is an eight-dimensional quasi-projective scheme $T_{\Sigma}$ fitting into a diagram

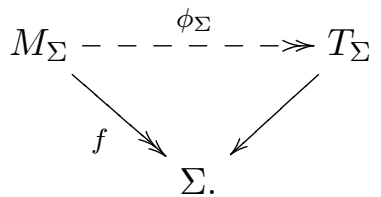

The fibers of $\phi_{\Sigma}$ are rational constant cycle curves in $M$.

Proof A general point in $M_{\Sigma}$ is the pushforward of a line bundle on a reducible curve $i: D \hookrightarrow S$ and by the combination of Theorem 3.2 and Lemma 3.6(ii) the class $\left[i_{*} \mathcal{L}\right] \in \mathrm{CH}_{0}(M)$ depends exactly on the restriction of $\mathcal{L}$ to each component. This is, what $T_{\Sigma}$ shall parameterize.

Let $\mathcal{C}_{\Sigma \backslash \Delta} \rightarrow \Sigma \backslash \Delta$ be the universal curve over $\Sigma \backslash \Delta$. Even though every fiber has two irreducible components, the total space $\mathcal{C}_{\Sigma \backslash \Delta}$ is irreducible. However, after the base change

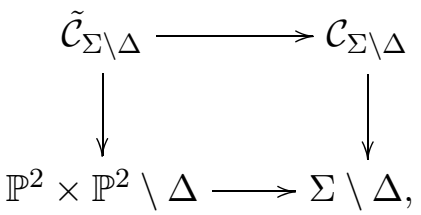

we have a decomposition $\tilde{\mathcal{C}}_{\Sigma \backslash \Delta}=\tilde{\mathcal{C}}_{\Sigma \backslash \Delta}^{1} \cup \tilde{\mathcal{C}}_{\Sigma \backslash \Delta}^{2}$ into two irreducible components, which are identified under the natural $\mathbb{Z} / 2 \mathbb{Z}$-action. Note that the horizontal arrows are principal $\mathbb{Z} / 2 \mathbb{Z}$-bundles and the vertical arrows are $\mathbb{Z} / 2 \mathbb{Z}$-equivariant. On the level of Picard schemes, restricting to each component gives a $\mathbb{Z} / 2 \mathbb{Z}$-equivariant map

$$
\operatorname{Pic}_{\tilde{\mathcal{C}}_{\Sigma \backslash \Delta} / \Sigma \backslash \Delta} \longrightarrow \operatorname{Pic}_{\tilde{\mathcal{C}}_{\Sigma \backslash \Delta}^{1} / \Sigma \backslash \Delta} \times_{\Sigma \backslash \Delta} \operatorname{Pic}_{\tilde{\mathcal{C}}_{\Sigma \backslash \Delta}^{2} / \Sigma \backslash \Delta}
$$

Here, $\mathbb{Z} / 2 \mathbb{Z}=\langle\tau\rangle$ acts on the right hand side via

$$
\tau \cdot\left(\mathcal{L}_{1}, \mathcal{L}_{2}\right)=\left(\tau^{*} \mathcal{L}_{2}, \tau^{*} \mathcal{L}_{1}\right) .
$$


(By a slight abuse of notation, $\tau$ also denotes the map identifying the isomorphic components $\tilde{\mathcal{C}}_{\Sigma \backslash \Delta}^{1}$ and $\tilde{\mathcal{C}}_{\Sigma \backslash \Delta}^{2}$ ). The quotient

$$
\operatorname{Pic}_{\tilde{\mathcal{C}}_{\Sigma \backslash \Delta} / \Sigma \backslash \Delta} / \tau \cong \operatorname{Pic}_{\mathcal{C}_{\Sigma \backslash \Delta} / \Sigma \backslash \Delta} \longrightarrow\left(\operatorname{Pic}_{\tilde{C}_{\Sigma \backslash \Delta}^{1} / \Sigma \backslash \Delta} \times_{\Sigma \backslash \Delta} \operatorname{Pic}_{\tilde{\mathcal{C}}_{\Sigma \backslash \Delta}^{2} / \Sigma \backslash \Delta}\right) / \tau
$$

of (4.3) by $\tau$ is what we are looking for, when restricted to the component, where the correct degree has been fixed. More precisely, we set

$$
T_{\Sigma}:=\left(\operatorname{Pic}_{\tilde{\mathcal{C}}_{\Sigma \backslash \Delta}^{1} / \Sigma \backslash \Delta}^{1} \times_{\Sigma \backslash \Delta} \operatorname{Pic}_{\tilde{\mathcal{C}}_{\Sigma \backslash \Delta}^{2} / \Sigma \backslash \Delta}^{2} \sqcup \operatorname{Pic}_{\tilde{\mathcal{C}}_{\Sigma \backslash \Delta}^{1} / \Sigma \backslash \Delta}^{2} \times_{\Sigma \backslash \Delta} \operatorname{Pic}_{\tilde{\mathcal{C}}_{\Sigma \backslash \Delta}^{2} / \Sigma \backslash \Delta}^{1}\right) / \tau
$$

and take $\phi_{\Sigma}$ to be the above map, whose fibers are isomorphic to $\mathbb{G}_{m}$.

Proposition 4.7 There is a four-dimensional quasi-projective scheme $T_{\Delta}$ fitting into a diagram

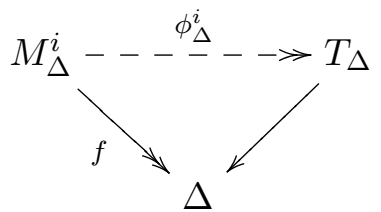

for $i=0,1$. The fibers of $\phi_{\Delta}^{0}$ are three-dimensional rational constant cycle subvarieties in M. Over $2 C \in \Delta$, the fibers of $\left(\phi_{\Delta}^{1}\right)_{2 C}$ are birational to a $\mathbb{P}^{2}$-bundle over a curve of genus 17 that is étale of degree 16 over $C$. In particular, they yield examples of three-dimensional constant cycle subvarieties in $M$ that are not rationally connected.

Proof We consider the component $M_{\Delta}^{0}$ first. A general point in $M_{\Delta}^{0}$ is of the form $\mathcal{E}=i_{*} \mathcal{E}_{0}$, where $i: C \hookrightarrow S$ is the inclusion of a smooth curve $C \in|H| \cong \Delta$ and $\mathcal{E}_{0}$ is a vector bundle of rank 2 on $C$. Again, by Theorem 3.2 and Example 3.7(iii) the class $\left[i_{*} \mathcal{E}_{0}\right] \in \mathrm{CH}_{0}(M)$ is determined by $i_{*} c_{1}\left(\mathcal{E}_{0}\right)$. This suggests to set

$$
T_{\Delta}:=\operatorname{Pic}_{\mathcal{C}_{U} / U}^{1},
$$

where $U \subset|H|$ is the open subset of smooth curves and $\mathcal{C}_{U} \rightarrow U$ denotes the universal curve and then define $\phi_{\Delta}^{0}$ as the determinant map. The fibers of $\phi_{\Delta}^{0}$ are isomorphic to a moduli space of stable vector bundles of rank two with fixed determinant of degree one, which is rational [25, 26, Proposition 2].

To deal with $M_{\Delta}^{1}$, let $\mathcal{E} \in M_{\Delta}^{1} \backslash\left(M_{\Delta}^{0} \cap M_{\Delta}^{1}\right)$ such that $C:=\operatorname{Supp}(\mathcal{E})_{\text {red }} \in U$. Then, by (2.8) and Lemma 3.6 the class $\operatorname{ch}_{2}(\mathcal{E})$ is determined by $i_{*} c_{1}\left(\mathcal{L}^{\otimes 2}(x) \otimes \omega_{C}^{-1}\right)$, where $\mathcal{L}:=\left.\mathcal{E}\right|_{C} / \mathcal{T} \in \operatorname{Pic}^{1}(C)$ and $x:=\operatorname{Supp}(\mathcal{T}) \in C$ with $\mathcal{T}$ being the torsion subsheaf of $\left.\mathcal{E}\right|_{C}$ and $i: C \hookrightarrow S$ being the inclusion. Consequently, we define

$$
\phi_{\Delta}^{1}: M_{\Delta}^{1} \rightarrow T_{\Delta}, \mathcal{E} \mapsto \mathcal{L}^{\otimes 2}(x) \otimes \omega_{C}^{-1} .
$$

We want to compute the fibers of $\left(\phi_{\Delta}^{1}\right)_{2 C}$. First, we forget the twist with $\omega_{C}^{-1}$. Then we can factor $\left(\phi_{\Delta}^{1}\right)_{2 C}$ as follows

$$
M_{2 C}^{1} \rightarrow \operatorname{Pic}^{1}(C) \times C \rightarrow \operatorname{Pic}^{3}(C), \mathcal{E} \mapsto(\mathcal{L}, x) \mapsto \mathcal{L}^{\otimes 2}(x) .
$$


The first arrow is defined outside the intersection $M_{2 C}^{0} \cap M_{2 C}^{1}$ and its fibers are a torsor under $\operatorname{Ext}_{C}^{1}\left(i_{*} \mathcal{L}, i_{*}\left(\mathcal{L}(x) \otimes \omega_{C}^{-1}\right)\right) \cong \mathbb{C}^{2}$, cf. [15, Corollary 3.5]. Thus the fibers of $\left(\phi_{\Delta}^{1}\right)_{2 C}$ are an $\mathbb{A}^{2}$-bundle over the fibers of the second arrow, which we factor as follows

$$
\operatorname{Pic}^{1}(C) \times C \rightarrow \operatorname{Pic}^{2}(C) \times C \stackrel{\mu}{\rightarrow} \operatorname{Pic}^{3}(C),(\mathcal{L}, x) \mapsto\left(\mathcal{L}^{\otimes 2}, x\right) \mapsto \mathcal{L}^{\otimes 2}(x) .
$$

Here, the first map is étale of degree 16 and the fibers of the second map

$$
\mu: \operatorname{Pic}^{2}(C) \times C \rightarrow \operatorname{Pic}^{3}(C),(\mathcal{L}, x) \mapsto \mathcal{L}(x)
$$

are isomorphic to $C$. To see this, let $\mathcal{M} \in \operatorname{Pic}^{3}(C)$ and consider $p_{2}: \mu^{-1}(\mathcal{M}) \rightarrow C$. As $\mathcal{L}(x) \cong \mathcal{M}$ for fixed $x \in C$ determines $\mathcal{L} \in \operatorname{Pic}^{2}(C)$, this projection is an isomorphism and the claim follows.

Remark 4.8 A combination of the proofs of Propositions 4.5-4.7 allows one to find the isotropic fibrations for the remaining cases $M_{V_{i}}, i=1,2,3$.

\subsection{More examples}

We construct some more examples of algebraically coisotropic subvarieties.

\subsubsection{Horizontal constant cycle Lagrangians}

To start with, we produce a constant cycle Lagrangian that dominates $B$. For example, any section of $M \rightarrow B$ would work. Unfortunately, $f$ does not admit a section [2]. Below, we produce a multisection of degree of $2^{10}$. Recall that

$$
M^{\circ} \cong \operatorname{Pic}_{\mathcal{C}^{\circ} / B^{\circ}}^{3},
$$

where $B^{\circ}=B \backslash\left(\Lambda_{4} \cup \Sigma\right)$ is the locus of smooth curves, and there is an exact sequence [12, $(9.2 .11 .5)]$

$$
0 \rightarrow \operatorname{Pic}\left(\mathcal{C}^{\circ}\right) / \operatorname{Pic}\left(B^{\circ}\right) \rightarrow \operatorname{Pic}_{\mathcal{C}^{\circ} / B^{\circ}}\left(B^{\circ}\right) \rightarrow \operatorname{Br}\left(B^{\circ}\right) \rightarrow \ldots
$$

Next, we claim that

$$
\operatorname{Pic}\left(\mathcal{C}^{\circ}\right) / \operatorname{Pic}\left(B^{\circ}\right) \cong \operatorname{Pic}(\mathcal{C}) / \operatorname{Pic}(B) \cong \operatorname{Pic}(S) \text {. }
$$

The first isomorphism follows from the diagram with exact rows and columns

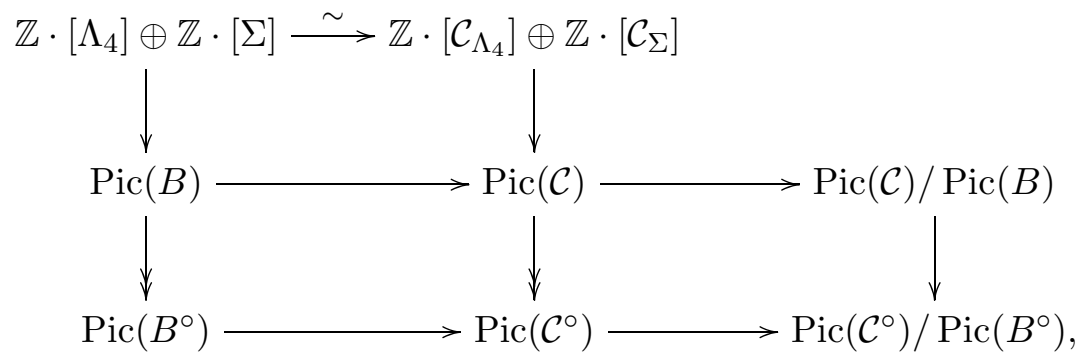


where we use, that the restriction of the universal curve over $\Lambda_{4}$ and $\Sigma$, respectively is irreducible. The second isomorphism in (4.5) holds, because $\mathcal{C} \subset B \times S$ is a $\mathbb{P}^{4}$-bundle over $S$ with $\mathcal{O}_{p_{2}}(1)=p_{1}^{*} \mathcal{O}_{B}(1)$. After this identification, any $L \in \operatorname{Pic}(S)$ with $n=2 H$. $L$ is mapped under the first morphism in (4.4) to the section

$$
s_{L}: B^{\circ} \rightarrow \operatorname{Pic}_{\mathcal{C}^{\circ} / B^{\circ}}^{n}, D \mapsto \text { L.D. }
$$

If Pic $(S)=\mathbb{Z} \cdot H$, for example, one gets sections for $n \equiv 0 \bmod 4$. There is always a section of $\mathrm{Pic}_{\mathcal{C}^{\circ} / B^{\circ}}^{2}$ that does not come from $S$.

Lemma 4.9 There is a section

$$
g_{2}^{1}: B^{\circ} \rightarrow \operatorname{Pic}_{\mathcal{C}^{\circ} / B^{\circ}}^{2}
$$

such that a curve $D \in B^{\circ}$ maps to the unique line bundle $g_{2}^{1}(D) \in \operatorname{Pic}^{2}(D)$ with $h^{0}\left(g_{2}^{1}(D)\right)=2$. In particular,

$$
\left(g_{2}^{1}\right) \otimes\left(g_{2}^{1}\right)=s_{H}: B^{\circ} \rightarrow \operatorname{Pic}_{\mathcal{C}^{\circ} / B^{\circ}}^{4}
$$

and $g_{2}^{1}$ is not of the form $s_{L}$ for $L \in \operatorname{Pic}(S)$.

Proof This is a consequence of the same phenomenon occurring for the universal family of smooth quadrics in $\mathbb{P}^{2}$. We identify $B=\left|\mathcal{O}_{\mathbb{P}^{2}}(2)\right|$ and we will see that the lemma holds true for $B^{\circ}=B \backslash \Sigma$. Let $\mathcal{Q}^{\circ} \subset B^{\circ} \times \mathbb{P}^{2}$ be the universal quadric, which is an étale $\mathbb{P}^{1}$-fibration, but not a projective bundle. We claim that there is a section

$$
s: B^{\circ} \rightarrow \operatorname{Pic}_{\mathcal{Q}^{\circ} / B^{\circ}}^{1}
$$

that is not induced by a line bundle on $\mathcal{Q}^{\circ}$. Indeed, fix a line $\ell \subset \mathbb{P}^{2}$ and consider

$$
\tilde{B^{\circ}}:=\mathcal{Q}^{\circ} \cap\left(B^{\circ} \times \ell\right) \rightarrow B^{\circ} .
$$

This morphism is finite, flat of degree 2 and the base change $\tilde{\mathcal{Q}}^{\circ} \rightarrow \tilde{B^{\circ}}$ admits a section. Therefore we get

$$
\tilde{s}: \tilde{B^{\circ}} \rightarrow \operatorname{Pic}_{\tilde{\mathcal{Q}}^{\circ} / \tilde{B}^{\circ}}^{1}
$$

As the two points in $\tilde{B^{\circ}}$ lying over a fixed point in $B^{\circ}$ define the same line bundle, $\tilde{s}$ descends to a section $s$. By definition, $s \otimes s$ is the section defined by $p_{2}^{*} \mathcal{O}_{\mathbb{P}^{2}}(1)$, which does not admit a square root. Pulling back $s$ along $\mathcal{C}^{\circ} \rightarrow \mathcal{Q}^{\circ}$ defines $g_{2}^{1}$.

Remark 4.10 In all our examples, it does not matter if we identify $M$ and $M_{H}(0,2 H,-3)$ via the isomorphism (4.2) (given by tensorization and dualization) or the birational map induced by the section $g_{2}^{1}$. The composition of the one map with the inverse of the other is the rational involution on $M$ that comes from the natural involution $\imath^{[5]}$ on $S^{[5]}$.

Now, we use the squaring map

$$
\rho_{2}: \operatorname{Pic}_{\mathcal{C}^{\circ} / B^{\circ}}^{1} \longrightarrow \operatorname{Pic}_{\mathcal{C}^{\circ} / B^{\circ}}^{2}, \mathcal{L} \mapsto \mathcal{L}^{\otimes 2}
$$

to construct a constant cycle Lagrangian from $g_{2}^{1}\left(B^{\circ}\right)$. Specifically, we set 


$$
L_{2}^{1}:=\overline{\rho_{2}^{-1}\left(g_{2}^{1}\left(B^{\circ}\right)\right)} \subset M_{H}(0,2 H,-3) \stackrel{\sim}{\longrightarrow} M .
$$

Lemma 4.11 The subvariety $L_{2}^{1}$ is a constant cycle Lagrangian in $M$, which is generically finite of degree $2^{10}$ over $B$.

Proof It is clear, that $\operatorname{dim} L_{2}^{1}=5$. We will show that $L_{2}^{1} \subset F_{0} M_{H}(0,2 H,-3)$. Let $D \in B^{\circ}$ and $\mathcal{L} \in \operatorname{Pic}^{1}(D)$ such that $\mathcal{L}^{\otimes 2}=g_{2}^{1}(D)$. Then

$$
4 \cdot i_{*} c_{1}(\mathcal{L})=i_{*} c_{1}\left(\left.\mathcal{O}_{S}(H)\right|_{D}\right)=4 c_{S} \in \mathrm{CH}_{0}(S) .
$$

This implies $i_{*} c_{1}(\mathcal{L})=c_{S}$ because $\mathrm{CH}_{0}(S)$ is torsionfree. We conclude that a general point in $L_{2}^{1}$ is contained in $S_{0} \mathrm{CH}_{0}(S)$ as desired. Finally, $\rho_{2}$ is finite, étale of degree $2^{10}$.

Remark 4.12 Another example of a horizontal constant cycle Lagrangian is constructed more generally for any Lagrangian fibration by Lin [21].

\subsubsection{Examples in $M_{\Delta}$}

Starting from $\phi_{\Delta}^{i}: M_{\Delta}^{i} \rightarrow T_{\Delta}$ (cf. Proposition 4.7), we construct two examples of constant cycle Lagrangians in $M_{\Delta}^{0}$ and $M_{\Delta}^{1}$. Recall that $T_{\Delta}=\operatorname{Pic}_{\mathcal{C}_{U} / U}^{1}$, where $U \subset|H|$ is the open subset consisting of smooth curves and the fibers of $\phi_{\Delta}^{i}$ are three-dimensional constant cycle subvarieties in $M$.

The idea of Example 4.13 is to find a constant cycle surface in $T_{\Delta}$. Then the preimage under $\phi_{\Delta}^{i}$ is a constant cycle Lagrangian in $M$ contained in $M_{\Delta}^{i}$. This idea is taken further in Example 4.14. Here, we find a surface in $T_{\Delta}$, that consists of line bundles whose first Chern class is a multiple of the Beauville-Voisin class, when pushed forward to $S$. By Theorem 3.5, the preimage of this surface is also a constant cycle Lagrangian in $M$.

For simplicity, we assume from now on that $\operatorname{Pic}(S)=\mathbb{Z} \cdot H$. Then every curve in $|H|$ is integral and $\mathrm{Pic}_{\mathcal{C}_{|H|} /|H|}^{1}$ is representable by a smooth, quasi-projective scheme.

Example 4.13 We construct a constant cycle subvariety of $\mathrm{Pic}_{\mathcal{C}_{|H|} /|H|}^{1}$ applying the same trick as for the construction of $L_{2}^{1}$. Namely, let

$$
\rho_{2}: \operatorname{Pic}_{\mathcal{C}_{|H|} /|H|}^{1} \rightarrow \operatorname{Pic}_{\mathcal{C}_{|H|} /|H|}^{2}
$$

and consider the section $s_{H}$ of $\operatorname{Pic}_{\mathcal{C}_{|H|} /|H|}^{2}$ defined by $H$. We set

$$
Z_{H}:=\rho_{2}^{-1}\left(s_{H}(|H|)\right) \text {. }
$$

Since Pic ${ }_{\mathcal{C}_{|H|} /|H|}^{2}$ can be embedded as an open subset of $M_{H}(0, H, 2)$, we can apply Theorem 3.5 to see that $Z_{H}$ is a constant cycle subvariety, as in the proof of Lemma 4.11. Now, $Z_{H} \subset \mathrm{Pic}_{\mathcal{C}_{|H|} /|H|}^{2}$ is a smooth, quasi-projective surface and the morphism $Z_{H} \rightarrow|H|$ is finite, étale of degree $2^{4}$, when restricted to the open subset of smooth curves $U \subset|H|$. By Lemma 2.2, we know that $U=|H| \backslash V(1,|H|)$ is the complement of a nodal curve of degree 30. Therefore $\pi_{1}(U) \cong \mathbb{Z} / 30 \mathbb{Z}\left[10\right.$, Proposition 1.3 and Theorem 1.13]. Consequently, $Z_{H}$ must have 8 pairwise isomorphic connected components that restrict over $U$ to the unique degree 2 cover of $U$. We replace $Z_{H}$ by one of its irreducible components and define 


$$
L^{i}: \overline{\left(\phi_{\Delta}^{i}\right)^{-1}\left(Z_{H}\right)} \subset M_{\Delta}^{i} \text { for } i=0,1 .
$$

By construction, these are constant cycle Lagrangians in $M$.

Example 4.14 The idea of this example is to consider the preimage of two-dimesional subvarieties in $T_{\Delta}$ that are not constant cycle subvarieties themselves, but consist of line bundles whose first Chern class is the Beauville-Voisin class when pushed forward to $S$.

To begin with, we have an embedding

$$
\Theta: \mathcal{C}_{|H|} \hookrightarrow \operatorname{Pic}_{\mathcal{C}_{|H|} /|H|}^{1}, C \ni x \mapsto \mathcal{O}_{C}(x) .
$$

Then, for example a vector bundle $\mathcal{E} \in M_{\Delta}^{0}$ lies over $\Theta\left(\mathcal{C}_{|H|}\right)$ if and only if its determinant line bundle is effective (of degree one). Therefore,

$$
\left(\phi_{\Delta}^{i}\right)^{-1}\left(\Theta\left(\mathcal{C}_{|H|}\right)\right) \subset F_{1} M \text { and } \operatorname{codim}\left(\phi_{\Delta}^{i}\right)^{-1}\left(\Theta\left(\mathcal{C}_{|H|}\right)\right)=4 .
$$

In particular, $\left(\phi_{\Delta}^{i}\right)^{-1}\left(\Theta\left(\mathcal{C}_{|H|}\right)\right)$ is algebraically coisotropic. The isotropic fibration is given by the composition of the projection $\mathcal{C} \rightarrow S$ with $\phi_{\Delta}^{i}$.

Refining this example yields constant cycle Lagrangians in $M_{\Delta}^{i}$ as follows. For example, let $C_{\mathrm{cc}} \subset S$ be a constant cycle curve and set

$$
L_{C_{\mathrm{cc}}}:=\left(\phi_{\Delta}^{i}\right)^{-1}\left(\Theta\left(\mathcal{C}_{|H|} \cap C_{\mathrm{cc}} \times|H|\right)\right) .
$$

\subsubsection{Examples in $S^{[5]}$}

We can also produce easily examples of algebraically coisotropic subvarieties in $S^{[5]}$. As $M$ and $S^{[5]}$ are birational, we have

$$
\mathrm{CH}_{0}\left(S^{[5]}\right) \cong \mathrm{CH}_{0}(M)
$$

[13, Example 16.1.11] and algebraically coisotropic varieties that are not contained in the exceptional locus of a birational map can be transferred from $S^{[5]}$ to $M$ and vice versa.

Example 4.15 This example can also be found in [32, §4 Example 1]. For $i=1, \ldots, 4$, define

$$
E_{i}:=\left\{\xi \in S^{[5]} \mid \text { length }\left(\mathcal{O}_{\xi_{\text {red }}}\right) \leq i\right\} .
$$

Then $E_{i} \subset S^{[5]}$ is closed subvariety of codimension $5-i[6]$. For example, $E:=E_{4}$ is the exceptional divisor of the Hilbert-Chow morphism $s: S^{[5]} \rightarrow S^{(5)}$. The irreducible components $E_{i}^{n}$ of $E_{i}$ are indexed by ordered tuples of positive natural numbers $\underline{n}=\left(n_{1} \geq n_{2} \geq \ldots \geq n_{i}\right)$ such that $\sum_{k=1}^{i} n_{k}=5$. In particular, $E_{4}$ and $E_{1}$ are irreducible, whereas $E_{3}$ and $E_{2}$ consists of two irreducible components. To sum up

$$
E_{1} \subset E_{2} \subset E_{3} \subset E_{4}=E \subset S^{[5]} .
$$

By definition of $E_{i}$ and Theorem 3.2, we have

$$
E_{i} \subset F_{i} S^{[5]}
$$


for all $i=1, \ldots, 4$ and hence $E_{i}$ is algebraically coisotropic.

Example 4.16 We have $\mathbb{P}^{2} \subset S^{[2]}$ given by $x \mapsto \pi^{-1}(x)$, where $\pi: S \rightarrow \mathbb{P}^{2}$. Consider the generically injective rational maps

$$
g_{3}: \mathbb{P}^{2} \times S^{[3]} \rightarrow S^{[5]} \text { and } g_{1}: \operatorname{Sym}^{2}\left(\mathbb{P}^{2}\right) \times S \rightarrow S^{[5]}
$$

and set

$$
P_{i}:=\overline{\operatorname{im}\left(g_{i}\right)} \subset S^{[5]} \text { for } i=1,3 .
$$

Clearly, $P_{i} \subset F_{i} S^{[5]}$ and $\operatorname{codim} P_{i}=5-i$.

Example 4.17 This example is taken from Knutsen et al. [19]. As in (2.2), we consider the locus $V(j,|H|) \subset|H|$ of curves $C$ with $g(\tilde{C}) \leq j$ for $j=0,1,2$. Specifically, $V(2,|H|)$ is everything, $V(1,|H|) \subset|H|$ is irreducible and one-dimensional and the generic curve in $V(1,|H|)$ has exactly one node, $V(0,|H|)$ is the discrete set of rational curves. We let $\mathcal{C}_{j} \rightarrow V(2-j,|H|)$ be the respective restriction of the universal curve $\mathcal{C}_{|H|} \rightarrow|H|$. For $2-j \leq i \leq 4$, consider the diagram

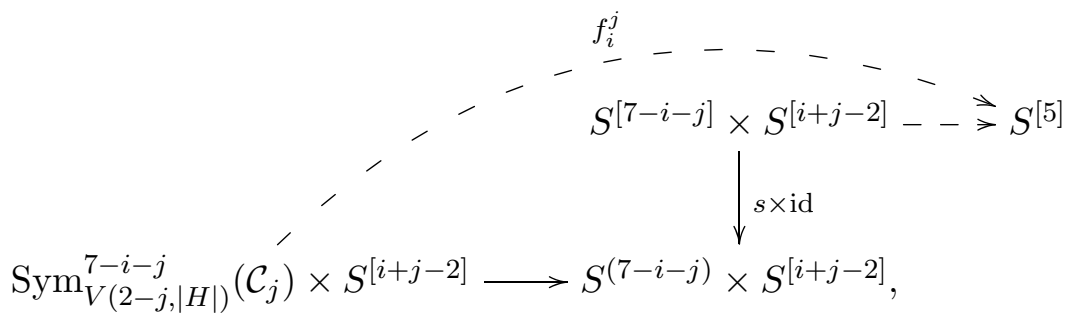

in which the lower horizontal map turns out to be generically injective [19, Theorem 6.4]. Hence, $f_{i}^{j}$ is generically finite. We define

$$
W_{i}^{j}: \overline{\operatorname{im}\left(f_{i}^{j}\right)} \subset S^{[5]} .
$$

This is a subvariety of codimension $5-i$, which is irreducible for $j \neq 2$. We have the following table of inclusions:

$$
\begin{array}{ccccccc}
W_{2}^{0} & \subset W_{3}^{0} & \subset W_{4}^{0} & & & \\
\cup & U^{1} & \cup & & & \\
W_{1}^{1} & \subset W_{3}^{1} & \subset W_{3}^{1} & \subset W_{4}^{1} & \\
\cup & U^{U} & \cup & \cup & \\
W_{0}^{2} & \subset & W_{1}^{2} & \subset W_{2}^{2} & \subset W_{3}^{2} & \subset W_{4}^{2} .
\end{array}
$$

A generic point $\xi \in W_{i}^{j}$ corresponds to a subscheme in $S$ that contains exactly $7-i-j$ points, which lie on a curve $C \in V(2-j,|H|)$ and the other $i+j-2$ points can move freely outside $C$. Hence, $[\xi] \in \mathrm{CH}_{0}(S)$ is contained in the $(2-j)+(i+j-2)$-th step of O'Grady's filtration. In other words,

$$
W_{i}^{j} \subset F_{i} S^{[5]}
$$

and $W_{i}^{j}$ is algebraically coisotropic. 
Remark 4.18 In [16, Proposition 4.4] it is shown that $W_{2}^{0}$ is the $\mathbb{P}^{3}$-bundle over $M_{H}(0, H,-6)$ parameterizing extensions $\operatorname{Ext}_{S}^{1}\left(\mathcal{E}, \mathcal{O}_{S}(-H)\right)$. Moreover, $W_{3}^{0} \backslash W_{2}^{0}$ has the structure of a $\mathbb{P}^{2}$ -bundle over a dense open subset of $S \times M_{H}(0, H,-5)$. This bundle parameterizes ideal sheaves $\mathcal{I} \in M_{H}(1,0,-4)=S^{[5]}$ that fit into an extension

$$
0 \rightarrow \mathcal{I}_{x}(-H) \rightarrow \mathcal{I} \rightarrow \mathcal{E} \rightarrow 0
$$

Acknowledgements This work is part of my PhD thesis at the University of Bonn. I'm extremely grateful to Daniel Huybrechts for all his time and good advice. I wish to thank Thorsten Beckmann, Giovanni Mongardi, Denis Nesterov, Georg Oberdieck, Giulia Saccà and Johannes Schmitt for their help. Additional and special thanks go to Hsueh-Yung Lin and Mirko Mauri! I thank the anonymous referee for the thorough reading of the manuscript.

Funding Open Access funding enabled and organized by Projekt DEAL.

Open Access This article is licensed under a Creative Commons Attribution 4.0 International License, which permits use, sharing, adaptation, distribution and reproduction in any medium or format, as long as you give appropriate credit to the original author(s) and the source, provide a link to the Creative Commons licence, and indicate if changes were made. The images or other third party material in this article are included in the article's Creative Commons licence, unless indicated otherwise in a credit line to the material. If material is not included in the article's Creative Commons licence and your intended use is not permitted by statutory regulation or exceeds the permitted use, you will need to obtain permission directly from the copyright holder. To view a copy of this licence, visit http://creativecommons.org/licenses/by/4.0/.

\section{References}

1. Arbarello, E., Cornalba, M., Griffiths, P., Harris, J.: The Geometry of Algebraic Curves I. Grundlehren der mathematischen Wissenschaften, vol. 267. Springer (1985)

2. Bakker, B.: A classification of Lagrangian planes in holomorphic symplectic varieties. J. Inst. Math. Jussieu 16(4), 859-877 (2017)

3. Beauville, A.: Systèmes hamiltoniens complètement intégrable associés aux surfaces K3. Symp. Math. vol. 32, pp. 25-31. Academic Press (1991)

4. Beauville, A., Voisin, C.: On the Chow ring of a K3 surface. J. Algebr. Geom. 13(3), 417-426 (2004)

5. Beauville, A.: On the splitting of the Bloch-Beilinson filtration. Algebraic Cycles and Motives (vol. 2), London Math. Soc. Lecture Notes, vol. 344, pp. 38-53. Cambridge University Press (2007)

6. Briancon, J.: Description de Hilb ${ }^{n} \mathbb{C}$, y. Invent. Math. 41, 45-89 (1977)

7. Bridgeland, T.: Stability conditions on K3 surfaces. Duke Math. J. 141(2), 241-291 (2008)

8. de Cataldo, M.A., Rapagnetta, A., Saccà, G.: The Hodge numbers of O'Grady 10 via Ngô strings (2019). arXiv: 1905.03217

9. Dedieu, T., Sernesi, E.: Equigeneric and equisingular families of curves on surfaces. Publ. Mat. 61, 175-212 (2017)

10. Dimca, A.: Singularities and topology of hypersurfaces. Universitext, Springer (1992)

11. Donagi, R., Ein, L., Lazarsfeld, R.: Nilpotent cones and sheaves on K3 surfaces. Contemp. Math. 207, 51-61 (1997)

12. Fantechi, B., Göttsche, L., Illusie, L., Kleiman, S.L., Nitsure, N.: Fundamental algebraic geometry, Grothen-Dieck's FGA explained. In: Mathematical Surveys and Monographs, vol. 125. AMS (2005)

13. Fulton, W.: Intersection theory. In: Ergebnisse der Mathematik und ihrer Grenzgebiete, 2nd edn., p. 3. Folge. Springer, Berlin (1998)

14. Hartshorne, R.: Algebraic geometry. In: Graduate Texts in Mathematics, vol. 52. Springer (1977)

15. Hellmann, I.: The nilpotent cone in the Mukai system for rank two and genus two. Math. Ann. 380(3), 1687-1711 (2021)

16. Hellmann, I.: Birational geometry of the Mukai system of rank two and genus two (2020). arXiv:2009. 00489

17. Huybrechts, D., Lehn, M.: The geometry of moduli spaces of sheaves. In: Cambridge Mathematical Library, 2nd edn. Cambridge University Press (2010) 
18. Huybrechts, D.: Curves and cycles on K3 surfaces. Algebr. Geom. 1, 69-106 (2014)

19. Knutsen, A., Lelli-Chiesa, M., Mongardi, G.: Wall divisors and algebraically coisotropic subvarieties of irreducible holomorphic symplectic manifolds. Trans. Am. Math. Soc. 371, 1403-1438 (2019)

20. Le Potier, J.: Faisceaux semi-stables de dimension 1 sur le plan projectif. Rev. Roum. Pures Appl. 38(7-8), 635-678 (1993)

21. Lin, H.-Y.: Lagrangian constant cycle subvarieties in Lagrangian fibrations. Int. Math. Res. Not. No.1, 14-24 (2020)

22. Marian, A., Zhao, X.: On the group of zero-cycles of holomorphic symplectic varieties. Épij. Géom. Algébr. 4, 3 (2020)

23. Mukai, S.: Symplectic structure of the moduli space of sheaves on an abelian or K3 surface. Invent. Math. 77(1), 101-116 (1984)

24. Mumford, D.: Rational equivalence of 0-cycles on surfaces. J. Math. Kyoto Univ. 9, 195-204 (1968)

25. Newstead, P.E.: Rationality of moduli spaces of stable bundles. Math. Ann. 215, 251-268 (1975)

26. Newstead, P.E.: Correction to 'Rationality of moduli spaces of stable bundles'. Math. Ann. 249, 281$282(1980)$

27. O'Grady, K.: Moduli of sheaves and the Chow group of K3 surfaces. J. Math. Pures Appl. (9) 100(5), 701-718 (2013)

28. Sawon, J.: On the discriminant locus of a Lagrangian fibration. Math. Ann. 341, 201-221 (2008)

29. Shen, J., Yin, Q., Zhao, X.: Derived categories of K3 surfaces, O'Grady's filtration, and zero-cycles on holomorphic symplectic varieties. Compos. Math. 156(1), 179-197 (2020)

30. Voisin, C.: Hogde theory and complex algebraic geometry II. In: Cambridge Studies in Advanced Mathematics, vol. 77. Cambridge University Press (2003)

31. Voisin, C.: Rational equivalence of 0-cycles on K3 surfaces and conjectures of Huybrechts and O'Grady. In: Recent Advances in Algebraic Geometry, London Mathematical Society Lecture Notes Series, vol. 417, 422-436. Cambridge University Press (2015)

32. Voisin, C.: Remarks and questions on coisotropic subvarieties and 0-cycles of hyper-Kähler varieties. In: "K3 Surfaces and Their Moduli", Proceedings of the Schiermonnikoog conference 2014, Progress in Math, vol. 315, pp. 365-399. Birkhäuser (2016)

Publisher's Note Springer Nature remains neutral with regard to jurisdictional claims in published maps and institutional affiliations. 